Chirurg 2018 $89: 391$

https://doi.org/10.1007/s00104-018-0606-5

Online publiziert: 2. Februar 2018

(c) Springer Medizin Verlag $\mathrm{GmbH}$, ein Teil von Springer Nature 2018

CrossMark

Khosro Hekmat ${ }^{1}$ Christiane J. Bruns ${ }^{2}$

'Klinik und Poliklinik für Herz- und Thoraxchirurgie, Universität zu Köln, Köln, Deutschland

${ }^{2}$ Klinik und Poliklinik Allgemein-, Viszeral- und Tumorchirurgie, Universität zu Köln, Köln, Deutschland

\title{
Lobektomie vs. Radiotherapie bei Lungenkrebspatienten
}

\section{Originalpublikation}

Cornwell et al (2018) Video-assisted thoracoscopic lobectomy is associated with greater recurrence-free survival than stereotactic body radiotherapy for clinical stage I lung cancer. J Thorac Cardiovasc Surg 155(1):395-402. https://doi.org/10.1016/j. jtcvs.2017.07.065

Hintergrund. Die stereotaktische ablative Radiotherapie (SABR) hat sich zur Standardbehandlung für medizinisch inoperable Patienten im Frühstadium eines nichtkleinzelligen Bronchialkarzinoms (NSCLC) etabliert. Jüngste Daten deuten darauf hin, dass bei operablen Patienten die Ergebnisse der SABR mit denen der chirurgischen Resektion vergleichbar wären. Die Studie von Chang et al. in Lancet Oncology [1] aus dem Jahre 2015 hat insbesondere dazu beigetragen, dass immer mehr operable Patienten einer SABR zugeführt werden. In einer vielversprechenden klinischen Studie verglichen Cornwell et al. die SABR mit der VATS(„video assisted thoracoscopic surgery")-Lobektomie und konnten eine Überlegenheit der chirurgischen Therapie zeigen.

Methoden. In der vorliegenden retrospektiven Studie untersuchten die Autoren 183 Patienten (94,0\% männlich) im klinischen Stadium I eines NSCLC, die in den Jahren 2009 bis 2014 einer SABR $(n=56)$ oder einer VATS-Lobektomie $(n=127)$ unterzogen worden waren. Die statistische Methode des „propensity matching" wurde verwendet, um vergleichbarere Gruppen zu identifizieren. Primäre Endpunkte waren die Tumorkontrolle und das rezidivfreie und lungenkrebsspezifische Überleben.
Ergebnisse. In der Gesamtkohorte waren die Patienten, die eine SABR erhielten, älter und hatten mehr Komorbiditäten als die VATS-Lobektomie-Patienten (Medianalter, 79,5 vs. 64 Jahre). Bei den 37 Propensity-gematchten Paaren betrug die 3-jährige Tumorkontrollrate nach SABR nur 54,3\%, während die VATSLobektomie-Patienten hier eine Rezidivfreiheit von $90,6 \%$ erzielten $(p=0,0038)$. Die 1-Jahres-, die 3-Jahres- und die rezidivfreie 3-Jahres-Überlebensrate betrug $89,2 \%, 52,9 \%$ und $38,5 \%$ nach SABR gegenüber $94,6 \%, 85,7 \%$ und $82,8 \%$ nach VATS-Lobektomie $(p<0,005$ für alle Prozentangaben).

Fazit des Reviews. Die Ergebnisse der vorliegenden Studie mit 183 Patienten zeigen eindrücklich, dass bei Patienten im Frühstadium eines NSCLC die VATSLobektomie deutliche Vorteile gegenüber der SABR aufweist. Die oben zitierte Studie von Chang et al. hatte zwei registrierte Studien zusammengefasst (STARS und ROSEL), bei der insgesamt nur 58 Patienten randomisiert entweder einer SA$\mathrm{BR}(n=31)$ oder chirurgischen Resektion $(n=27)$ unterzogen wurden. Die 3-Jahres-Überlebensrate zeigte einen signifikanten $(p=0,037)$ Unterschied zugunsten der SABR (SABR: $95 \%$, Chirurgie: $79 \%)$. Die geringere Invasivität der SABR ist zunächst einmal als Vorteil für die Patienten zu werten. Dies sollte jedoch nicht auf Kosten einer erhöhten Rezidivrate erfolgen. Ob die SABR der operativen Resektion gleichwertig ist, muss durch weitere prospektive randomisierte Studien gezeigt werden. Die SABR ist eine geeignete Option für medizinisch inoperable Patienten, sollte jedoch zurzeit nicht bei operablen Patienten im Stadium I eines NSCLC angewandt werden.

\section{Korrespondenzadresse}

\section{Prof. Dr. K. Hekmat}

Klinik und Poliklinik für Herz- und

Thoraxchirurgie, Universität zu Köln

Kerpener Straße 62, 50931 Köln, Deutschland

khosro.hekmat@uk-koeln.de

Interessenkonflikt. K. Hekmat und C.J. Bruns geben an, dass kein Interessenkonflikt besteht.

\section{Literatur}

1. Chang et al (2015) Stereotactic ablative radiotherapy versus lobectomy for operable stage I non-small-cell lung cancer: a pooled analysis of two randomised trials. Lancet Oncol 16:630-637. https://doi.org/10.1016/S1470-2045(15)70168-3 